\section{Pathology of fore and midgut neuroendocrine tumours}

\author{
Salvador J Diaz-Cano
}

\begin{abstract}
There is much confusion in both definition and practical issues among pathologists and clinicians alike for neuroendocrine tumours/carcinomas (NETs/NECs).

This review focuses the attention on key issues of foregut and midgut NET: pathological features (nomenclature, classification, diagnostic criteria, grading, staging, markers and prognosis), molecular genetics, and how to approach common problems in NET (multifocal vs multicentric, metastatic potential and prediction of primary site).

The value of the term neuroendocrine is related to its connotation of a particular phenotype or differentiation pattern. Accordingly, the NET nomenclature can be addressed by using any of the following groups of terms: (1) well-differentiated NET, well-differentiated NEC, or poorly differentiated NEC; or (2) NECS (grades I-III), indicating in an explanatory note the equivalent terminology, when appropriate. Ultimately, the use of specific NET terms remains a personal preference, but what is the most critical is the necessity that the terms will be understood by health professionals caring for patients and that the terms can be grouped and translated for epidemiologic and molecular studies that can offer unique targets for specific therapies.
\end{abstract}

Keywords foregut; gastrointestinal tract; midgut; molecular markers; neuroendocrine tumour; pathology

\section{Brief statement on paper impact}

Although neuroendocrine tumours (NETs) are relatively rare and they have been the object of numerous investigations, there is much confusion in its classification and definition by health professionals. This review focuses the attention on key issues of foregut and midgut NET: general pathological features (nomenclature, classification, diagnostic criteria, general grading, staging, markers and prognosis), molecular genetics of sporadic and familiar NETs, and how to approach common problems in NET (synchronic vs metachronic neoplasms, metastatic potential and prediction of primary site).

Ultimately, the use of specific terms for these neoplasms remains a personal preference, but what is the most critical is the necessity that the terms will be understood by health

\footnotetext{
Salvador J Diaz-Cano MD PhD FRCPath is a Consultant Pathologist, Reader in Cellular and Molecular Pathology, Department of Histopathology, King's College Hospital and King's Health Partners, London, UK. Conflicts of interest: none.

Abbreviations: DNES, diffuse neuroendocrine system; GI, gastrointestinal; LOH, loss of heterozygosity; NE, neuroendocrine; NEC, neuroendocrine carcinoma; NET, neuroendocrine tumours; PD-NEC, poorly differentiated neuroendocrine carcinoma; WD-NEC, well-differentiated neuroendocrine carcinoma; WD-NET, well-differentiated neuroendocrine tumour.
}

professionals caring for patients and that the terms can be grouped and translated for epidemiologic studies.

\section{Introduction}

The endocrine cells scattered throughout practically all organs with an epithelial lining constitute the so-called diffuse neuroendocrine system (DNES) that shares common biochemical and pathological properties. Currently, the DNES concept incorporates the 1970's ideas of Pearse, the paraneuron concept of Fujita, and observations of other investigators who have contributed to the development and evolution of an endocrine system that is not limited to a particular organ and stores its secretion in membrane-bound cytoplasmic granules. Although these cells and their tumours have the object of numerous investigations throughout the years, there is much confusion in both definition and practical issues among pathologists and clinicians alike.

Neuroendocrine tumours (NET) of the gastrointestinal (GI) tract have been classically classified according to embryological considerations. ${ }^{1}$ Williams and Sandler proposed (1963) an embryologic classification of NETs based on their origins from foregut (stomach, duodenum, upper jejunum, and pancreas), midgut (lower jejunum, ileum, appendix, and cecum), and hindgut (colon and rectum) derivatives and demonstrated characteristic morphologic, histochemical, and immunohistochemical differences among the three groups. This classification offers correlation between the embryologic origin and the histologic pattern, argentaffin and diazo reaction, 5-hydroxy-tryptamine tumour content, urinary 5-hydroxy-indoleacetic acid, association with carcinoid syndrome, and metastasis to bone and skin. However, in the case of the foregut tumours, the usefulness of such a classification in practical diagnostic work is limited by its failure to characterize individual tumour entities with welldefined histological, hormonal, and/or clinicopathological profiles. After a brief embryological introduction, the present review will focus the attention on key issues of foregut and midgut NET: general pathological features (nomenclature, classification, diagnostic criteria, general grading, staging, markers and prognosis), molecular genetics of sporadic and familiar NE neoplasms, and how to approach common problems in NET (synchronic vs metachronic neoplasms, metastatic potential and prediction of primary site). Due to their special considerations, both lung and pancreatic NETs are not included in this review, except for specific issues related with its distinction from GI-NET.

\section{Embryological and anatomical considerations}

The story of the diffuse neuroendocrine system (DNES) started with the histological identification of chromaffin cells at the base of the normal bowel crypts by Kultschitsky (1897) and, pathologically, with the description of a peculiar little tumour of the small bowel and appendix with the terms of small carcinoma and carcinoid tumour by Lubarsch (1888) and Oberndorfer (1907), respectively. These cells were later revealed to share important biochemical pathways, symbolized by Pearse by the acronym APUD (Amine Precursor Uptake Decarboxylation), ${ }^{2}$ which was suggested to express a common origin from the neural crest (a transient embryonal neural structure located at the junction of the neural tube and the dorsal ectoderm) already known to be the progenitor of autonomic ganglia and plexuses, paraganglia, and 
melanocytes. ${ }^{3}$ This theory was undermined by more rigorous experiments, in particularly the ingenious quail-chick chimeric model devised by LeDouarin (1974).

Currently, only ganglia, paraganglia, melanocytes, and thyroid $\mathrm{C}$ cells are considered neural crest derivatives, ${ }^{3}$ while the other NE cells derive from the same local epithelial stem cells that give rise to all other epithelial cell types of the mucosa where these cells are located, ${ }^{4}$ as Cheng and Leblond proposed for the small bowel mucosa (1974). ${ }^{5}$ As a consequence, a substantial change in terminology has called into question the notions of NE cells and tumours. It has been proposed to drop the qualifier neuro from cells of non-neural derivations, and to call their tumours simply as endocrine; the pancreas is the best example of this approach, as sanctioned by the current WHO classification. Carrying this argument further, how can we explain the expression of thyroid transcription factor 1 by C-cell tumours or the existence of mixed follicular/papillary-medullary carcinomas? Although the current neurophobic tendency is true for certain locations, the neural crest derivation and the expression of neural markers are still valid. Despite these biological questions, the acronym NET is widely accepted and used clinically.

\section{General pathological features}

NETs occur in virtually all tissues and organs, including those that do not normally contain NE cells, and they may also occur as components of teratomas. These tumour cells, like their normal counterparts in the GI tract, express several antigens that are commonly expressed by neuronal elements and are commonly referred to as neuroendocrine markers (see this section below), independent of hormone production. It is for this reason that neuroendocrine is the preferred designation and the term NET is used in this review.

Specific trends in incidence for NETs of certain sites were identified, with a significant increase in the reported annual ageadjusted incidence of NETs from 1973 to 2004. The most common primary tumour site varied by race, with the lung being the most common in white patients, and the rectum being the most common in Asian/Pacific Islander, American Indian/Alaskan Native, and African American patients. ${ }^{6}$ Among the most recently collected subset of data, sites that demonstrated the greatest NET incidence were the gastrointestinal tract $(67.5 \%)$ and the bronchopulmonary system $(25.3 \%)$. Within the gastrointestinal tract, most NETs occurred in the small intestine $(41.8 \%)$, rectum (27.4\%), and stomach (8.7\%). For all sites, age-adjusted incidence rates were highest in black males ( 4.48 per 100,000 per year). The best 5 -year survival rates were recorded for patients with rectal (88.3\%), bronchopulmonary (73.5\%), and appendiceal $(71.0 \%)$ carcinoids; these tumours exhibit invasive growth or metastatic spread in $3.9 \%, 27.5 \%$, and $38.8 \%$ of patients, respectively. These findings bring into question the widely promulgated relative benignity of carcinoid disease. Certain NETs, such as those of the rectum, appear to be over-represented among the black and Asian populations within the United States, suggesting the role of genetics in the development of this intriguing disease. ${ }^{7}$

\section{Nomenclature and terminology}

The term karzinoid (carcinoid) meaning carcinoma-like was introduced by Oberndorfer to describe peculiar small intestine tumours that resembled cancers but had unusual clinical behaviour. This term has been applied differently by pathologists and clinicians: pathologists have traditionally classified welldifferentiated endocrine tumours of the lung, gut and pancreas as "carcinoid tumours", while clinicians use the term to describe the syndrome caused by serotonin excess. It has also become apparent that "carcinoid tumours" in different locations within the GI tract are not necessarily equivalent and that they can display the full histopathological spectrum from very low-grade to high-grade malignancy. For these reasons the term "carcinoid" has been increasingly discouraged in favour of more precise terminology.

NETs may be associated with clinical syndromes due to the overproduction of biologically active amines or peptide hormones, while many others may be clinically silent. In the latter instances, amines or peptides often are demonstrable by immunohistochemical or other techniques. There is extensive overlap with pancreatic endocrine tumours, for example, somatostatin-producing cells are present in both, and during development gastrin is produced in the pancreas, so that any discussion of GI-NETs has implications for pancreatic NETs.

Before the advent of immunohistochemical analysis, the NET diagnosis most often relied on the use of fixatives containing chromate salts, histochemical stains, or electron microscopic examination. Certain intestinal endocrine cells and tumours show a positive chromaffin reaction, similar to that observed in the adrenal medulla and paraganglia. Silver stains of both argentaffin (Masson-Fontana) and argyrophil (Grimelius, SevierMunger) types also were used, although these staining sequences often produced inconsistent results. Other stains that had been used for the detection of NE cells included lead haematoxylin and toluidine blue or coriophosphine $\mathrm{O}$ following acid hydrolysis (masked metachromasia). With the exception of the Grimelius method, these stains are now used rarely in the workup of NETs. Electron microscopic examination was used extensively in the past to demonstrate secretory granules, but this approach has been largely replaced by immunohistochemical studies.

\section{Classification}

Given the wide array of NE cells, it is not surprising the lack of unified classification. The NET categorization is based on tumour size, angioinvasion, extent of organ-specific invasion, proliferation index, functional status/hormonal syndrome, and metastases to lymph nodes or liver. ${ }^{8}$ Using these criteria, a siteindependent NET classification system (Figure 1) considers $^{9,10}$ :

- Well-differentiated NET (WD-NET)

○ Benign

- Uncertain malignant potential

- Well-differentiated neuroendocrine carcinoma (WDNEC) - low-grade malignant

- Poorly differentiated neuroendocrine carcinoma (PDNEC) - high-grade malignant

Well-differentiated, slowly growing GI-NETs and carcinomas, those that are also called carcinoids, which comprise a number of well-defined entities (e.g. gastrinomas, and others), are distinguished on the basis of their localization as well as their morphological and functional features. PD-NECs are composed of cells displaying high mitotic and Ki-67 indices, and few secretory granules, form a separate group not difficult to 


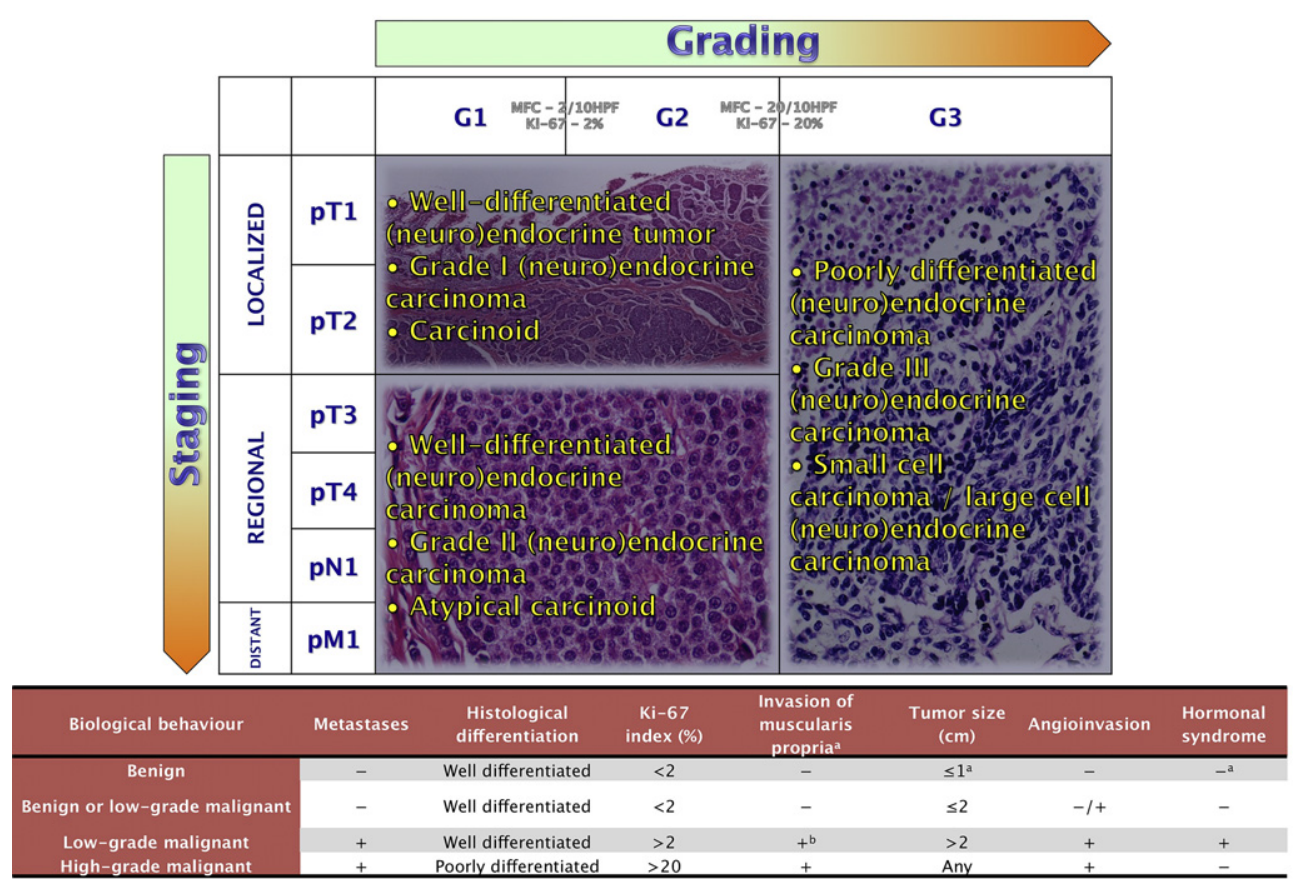

Figure 1 Grading and staging system proposed for NETs. Growth patterns, nuclear atypia, presence of necrosis and proliferation index are the main histological features for grading. These features appear closely related with the staging, which in the GI tract is determined by the depth of invasion and size of the neoplasm. More details are shown in Table. ${ }^{a}$ Exception: malignant duodenal gastrinomas are usually smaller than $1 \mathrm{~cm}$ and confined to the

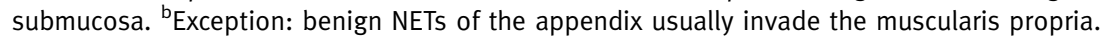

recognize as high-grade malignancies. However, an application of this classification has not resolved two issues: (a) site-specific variations and, therefore, the prediction of malignant behaviour. (b) Tissue- or site-specific distribution of certain NE cell types (Tables 1 and 2). ${ }^{11}$ Histamine-producing enterochromaffin-like cells are found only in the stomach; gastrin-producing NETs occur in the gastric antrum, upper intestine, and pancreas; and serotonin-producing enterochromaffin cells are distributed throughout the GI tract and pancreas, although tumours of these cells are most commonly encountered in the ileum and appendix.

\section{Diagnostic criteria. grading and staging}

The morphological appearance remains as the diagnostic gold standard. Undisputable malignancy criteria of WD-NETs are gross tumour invasion beyond the organ limits and/or metastases. Figure 1, based on the World Health Organization (WHO) criteria, provides checklists that allow an appropriate classification of an individual GI-NET. This tumour family contains synaptophysin as a common marker and can be further divided into epithelial (cytokeratin-positive) and neural (neurofilament-positive) subtypes on the basis of the intermediate filament profile.

NETs of the epithelial type exhibit a wide range of histologic and cytologic features (Figure 2). Common patterns include the presence of solid or nested typical of midgut tumours pattern to trabecular/gyriform, glandular, tubular-acinar, and mixed patterns more frequently encountered in foregut NET nests (insulae), ribbons, festoons, glands, and rosettes. Less commonly, cystic, papillary, pseudopapillary, fascicular, and socalled angiomatoid or angiomatous patterns may also be seen. Poorly differentiated lesions frequently reveal areas of necrosis (multifocal or confluent), diffuse or expansile growths and spindle cell differentiation. The cells may be round, polyhedral, or spindle shaped, and cell processes may be evident, particularly following the use of histochemical or immunohistochemical stains. Individual cells may be small, intermediate, or large and the cytoplasm may be granular, eosinophilic or amphophilic, oncocytic, or vacuolated as the result of the accumulation of mucin (amphicrine) or lipid droplets. Rarely, the cytoplasm may contain melanin granules. The nuclei may be small and hyperchromatic or intermediate to large with coarsely granular chromatin (salt and-pepper appearance). Nucleoli may be prominent in some tumour types. Ancillary findings include intracytoplasmic hyaline globules, nuclear pseudoinclusions, and associated amyloidosis. The presence of calcification is sometimes noted and, when psammomatous in nature in duodenal NET, is diagnostic of a duodenal somatostatin-producing NET. Where the diagnosis may not readily be apparent is when there is a cytologic variation. The constituent cells may have abundant granular eosinophilic cytoplasm because of accumulation of mitochondria resulting in an oncocytic/oxyphilic appearance, or the tumour cells may be spindle shaped, have a clear or finely vacuolated cytoplasm, or be large and pleomorphic sometimes with multiple nuclei or so-called rhabdoid. The latter cell has a characteristic appearance that is due to collapse of the cytoskeleton (misfolded proteins) with formation of perinuclear aggresomes composed of keratin filaments, often displace the nucleus peripherally, and appear as deeply eosinophilic cytoplasmic globules. Clear cell change may be seen focally in oncocytic lesions, or may be diffuse due to extreme glycogen or lipid accumulation. Another morphologic variant is a NET with ductules. This is not a mixed neuroendocrine-epithelial tumour but a NET with ductules that are either entrapped as the tumour 
Site-specific distribution of neuroendocrine cells of the GI tract

\begin{tabular}{|c|c|c|c|}
\hline Location & Cell type & Hormone/chemical & Function \\
\hline \multirow[t]{3}{*}{ Entire $\mathrm{Gl}$ tract } & Delta (D) & Somatostatin (SST) & $\begin{array}{l}\downarrow \text { Gastrin release } \\
\uparrow \text { Fluid absorption } \\
\uparrow \text { Smooth muscle contraction } \\
\downarrow \text { Endo/exocrine secretion } \\
\downarrow \text { Bile flow }\end{array}$ \\
\hline & $\begin{array}{l}\text { Enterochromaffin } \\
(E C)\end{array}$ & $\begin{array}{l}\text { Serotonin/substance P } \\
\text { /melatonin/guanylin }\end{array}$ & $\begin{array}{l}\text { SI and colon motility, possible vascular regulation } \\
\uparrow \text { Fluid secretion }\end{array}$ \\
\hline & Ghrelin (Gr) & Ghrelin & $\begin{array}{l}\text { Modulating appetite, adipogenesis, gut motility, glucose } \\
\text { metabolism, cell proliferation }\end{array}$ \\
\hline \multirow[t]{4}{*}{$\begin{array}{l}\text { Nerves (enteric } \\
\text { neurons) }\end{array}$} & Nerves & $\begin{array}{l}\text { Vasoactive intestinal peptide } \\
\text { (VIP) }\end{array}$ & $\begin{array}{l}\text { Smooth muscle contraction } \\
\uparrow S B \text { secretion } \\
\uparrow \text { Pancreatic secretion }\end{array}$ \\
\hline & Nerves & Substance $P$ & $\begin{array}{l}\uparrow \mathrm{GI} \text { motility } \\
\downarrow \mathrm{HCO} \text { secretion }\end{array}$ \\
\hline & Vagus nerve & Gastrin releasing protein (GRP) & Gastrin release \\
\hline & Nerves; SI, colon & Cholecystokinin (CKK) & $\uparrow$ Gallbladder contraction \\
\hline Gastric fundus & $\begin{array}{l}\text { Enterochromaffin- } \\
\text { like }(E C L)\end{array}$ & Histamine & +Gastric acid secretion \\
\hline $\begin{array}{l}\text { Gastric fundus \& } \\
\text { antrum }\end{array}$ & $x$ & $\begin{array}{l}\text { Islet amyloid polypeptide (IAPP, } \\
\text { Amylin) }\end{array}$ & Satiety regulation and inhibits gastric emptying \\
\hline $\begin{array}{l}\text { Gastric antrum \& } \\
\text { duodenum }\end{array}$ & G, IG & Gastrin & $\begin{array}{l}\uparrow \text { Acid secretion } \\
\uparrow \text { Motility }\end{array}$ \\
\hline \multirow[t]{3}{*}{ Duodenum } & I & Cholecystokinin (CCK) & $\begin{array}{l}\uparrow \text { Enzyme secretion } \\
\uparrow \text { Contraction }\end{array}$ \\
\hline & M & Motilin & Smooth muscle contraction (esoph, stom, duodenum) \\
\hline & $\mathrm{S}$ & Secretin & $\begin{array}{l}\uparrow \mathrm{HCO} 3 \text { and fluid secretion by pancreatic ducts } \\
\downarrow \text { Gastric a secretion }\end{array}$ \\
\hline Duodenum, jejunum & $\mathrm{K}$ & GIP & $\begin{array}{l}\downarrow \text { Fluid absorption } \\
\uparrow \text { Insulin release }\end{array}$ \\
\hline \multirow[t]{3}{*}{ Small intestine } & $\begin{array}{l}\text { PP } \\
\text { N (ileum) }\end{array}$ & $\begin{array}{l}\text { Pancreatic polypeptide (PP) } \\
\text { Neurotensin }\end{array}$ & $\begin{array}{l}\text { Pancreatic enzymes } \\
\downarrow \text { GI motility } \\
\uparrow \text { Blood flow }\end{array}$ \\
\hline & - & Enkephalin & $\begin{array}{l}\text { Opiate-like action } \\
\downarrow \text { Intestinal motility (constipation) }\end{array}$ \\
\hline & L (+colon) & $\begin{array}{l}\text { GLP-1 } \\
\text { Polypeptide YY (PYY) } \\
\text { Neuropeptide Y (NPY) }\end{array}$ & $\begin{array}{l}\text { Possible glucose regulation } \\
\downarrow \text { Vagal mediated acid secretion } \\
\downarrow \text { Enzyme and fluid secretion } \\
\text { Potent vasoconstrictor, inhibits ACh release }\end{array}$ \\
\hline Colon & - & PYY & $\begin{array}{l}\downarrow \text { Vagal mediated acid secretion } \\
\downarrow \text { Enzyme and fluid secretion }\end{array}$ \\
\hline
\end{tabular}

\section{Table 1}

grows into surrounding normal tissue or as a result of secondary reactive phenomenon.

NETs of epithelial type may occur in pure forms or may be admixed with non-endocrine components in the form of squamous carcinoma or adenocarcinoma. ${ }^{9}$ It is worth mentioning that the socalled goblet cell NETs of the appendix is a compositae or amphicrine carcinoma and not a pure NET, although it is included in many discourses on GI-NET. Such tumours with evidence of multidirectional or divergent differentiation are referred to as mixed endocrine-exocrine carcinomas. ${ }^{9}$ The presence of a neuroendocrine component in a non-endocrine tumour may be suggested on the basis of subtle histologic features and should be confirmed by immunohistochemistry or electron microscopy. The exact proportions of non-endocrine cells occurring within an endocrine tumour required for the diagnosis of a mixed exocrine-endocrine tumour is a matter of debate; only tumours showing balanced amounts of exocrine and endocrine components, whether present in single cells (amphicrine), separate but intimately admixed cells (combined tumours), or as separate tumour growths (compositae tumours), qualify for the diagnosis of mixed endocrine-exocrine carcinomas, with the same categories described in the NET classification above. ${ }^{9}$ Neuroendocrine cells also may be present as dispersed single cells or 
Site-specific distribution of neuroendocrine tumours of the GI tract

\begin{tabular}{|c|c|c|c|c|c|}
\hline Location & $\begin{array}{l}\text { Incidence } \\
\text { (\% GI-NET) }\end{array}$ & Types & Patterns & $\begin{array}{l}\text { Hormone } \\
\text { secretion }\end{array}$ & Comments \\
\hline Oesophagus & $0.05 \%$ & $\begin{array}{l}\text { WD - Barrett's } \\
\text { WD + Barrett's } \\
\text { PD NET }\end{array}$ & $\begin{array}{l}\text { WD - Trab/gl } \\
\text { WD - Trab/gl } \\
\text { PD - Solid }\end{array}$ & $\begin{array}{l}\text { No } \\
\text { ECL cells } \\
\text { ADH, VIP }\end{array}$ & \\
\hline Stomach & $2-4 \%$ & $\begin{array}{l}\text { Type } 1 \text { - CA } \\
\text { Type } 2 \text { - MEN1 } \\
\text { Type } 3-\text { WD } \\
\text { Type } 4-\text { PD }\end{array}$ & $\begin{array}{l}\text { WD }- \text { nested } \\
\text { WD }- \text { nested } \\
\text { WD }- \text { nested } \\
\text { PD }- \text { solid }\end{array}$ & $\begin{array}{l}\text { ECL cells } \\
\text { ECL cells } \\
\text { ECL/EC cells } \\
\text { No }\end{array}$ & $\begin{array}{l}\text { ECL hyperplasia } \\
\text { ECL nodules/tumours }\end{array}$ \\
\hline $\begin{array}{l}\text { Duodenum, } \\
\text { upper } \\
\text { jejunum }\end{array}$ & $20-25 \%$ & $\begin{array}{l}\text { Gastrin NET } \\
\text { SST NET } \\
\text { Gangliocytic PGL } \\
\text { WD gastrin NET } \\
\text { WD-NET }\end{array}$ & $\begin{array}{l}\text { WD - trab/gl } \\
\text { WD - gl/psam } \\
\text { WD - nested } \\
\text { WD - trab } \\
\text { WD - nested/trab }\end{array}$ & $\begin{array}{l}\text { Gastrin } \\
\text { SST } \\
\text { No } \\
\text { Gastrin } \\
\text { CT, } 5 \mathrm{HT}\end{array}$ & $\begin{array}{l} \pm \mathrm{ZES}, \mathrm{CDX}-2+\text { ve } \\
\pm \text { ChromA, } \pm \text { NF1 } \\
\text { S100 + ve sust cells, scattered GC } \\
\text { ZES, G cell hyperplasia, HP } \pm \text { PPI }\end{array}$ \\
\hline Jejunum-Ileum & $25 \%$ & $\begin{array}{l}\text { PD NET } \\
\text { WD-NET }\end{array}$ & $\begin{array}{l}\text { PD - solid } \\
\text { WD - Insular/crib }\end{array}$ & $\begin{array}{l} \pm \mathrm{SST} \\
5 \mathrm{HT}\end{array}$ & $\begin{array}{l}\text { Synapt + ve, ChromA -ve } \\
\text { EC cells, scattered S100 + ve cells, } \\
\text { CDX- } 2 \text { + ve, CEA + ve }(2 / 3)\end{array}$ \\
\hline Appendix & $20 \%$ & WD-NET & WD - Solid/islet-like & $5 \mathrm{HT}$ & EC cells, S100 +ve sust cells \\
\hline
\end{tabular}

\section{Table 2}

small cell groups in tumours arising in diverse anatomic sites, including metastatic deposits, especially in adenocarcinomas. ${ }^{9}$ In general, the presence of a minority of dispersed neuroendocrine cells in most non-endocrine tumours has no significant prognostic impact, although this point is far from settled.

NETS of the neural type include neuroblastomas and their mature congeners, paragangliomas and pheochromocytomas. Rarely, mixed paragangliomas and neuroblastomas may occur and have been referred to as compound or compositae tumours. Paragangliomas and pheochromocytomas, on the other hand, are characterized by a solid or nesting growth pattern. Individual cells are round, polyhedral, or spindle shaped with round to ovoid nuclei containing coarsely granular chromatin and usually a single distinct nucleolus. Cytoplasmic granularity often is apparent in well-fixed samples. Differentiation is manifested by the development of neuritic processes and, ultimately, by the formation of neurons. The least differentiated forms of neuroblastoma are characterized by the presence of small cells with round hyperchromatic nuclei.

In concert with the development of a reproducible classification system (the WHO classification), there has been considerable discussion for the formulation of a TNM staging and grading system under the aegis of the European Neuroendocrine Tumour Society. ${ }^{10,12,13}$ This TNM classification has been able to differentiate significantly between different tumour stages (stages I-III vs stage IV) and cellular proliferation rates according to Ki-67 labelling (grade 1 vs grade 2 ; grade 1 vs grade 3 and grade 2 vs grade 3). Cox regression analysis has confirmed an increased risk of reduced survival for patients with stage III or IV NET and grade 2 or 3 NET. Thus, the new classification system provides a valid and powerful tool for prognostic stratification of foregut NETs in clinical practice and research.

\section{Neuroendocrine markers}

Several markers of neuroendocrine differentiation and more cell-specific markers have emerged recently. The traditional baseline markers that have been the mainstay of NET workup are synaptophysin and chromogranin. Added to these are cellspecific markers and/or hormones that are relevant to the site of the NET. For instance, vasoactive monoamine transporter 2 is used for gastric enterochromaffin-like NET; and serotonin and substance P for ileal and appendiceal NET. Oftentimes, the functional status and the clinical symptoms of the patient will determine specific markers done in a particular case. Included in the baseline workup of pancreatic NET is Ki-67, as the Ki-67 index separates benign, WD-NET (2\%).

General markers include chromogranins/secretogranins, neuroendocrine secretory protein 55 (NESP-55), synaptophysin, synaptic vesicle protein 2 (SV2), hormonal profile, intermediate filaments, enzymes (including enolases, protein gene product 9.5, endopeptidases and carboxypeptidases like prohormone convertases and carboxypeptidases $\mathrm{H}$ and $\mathrm{E}$, histidine decarboxylase, catecholamine-synthesizing enzymes, somatostatin receptors (SSTR), and membrane and adhesion molecules (CD56, CD57, CD99) (Table 3). ${ }^{9,10}$

\section{Molecular genetics in NETs. Pathways in sporadic neoplasms and inherited syndromes}

Biologically, neoplasms develop through acquisition of capabilities that involve tumour cell aspects and modified microenvironment 


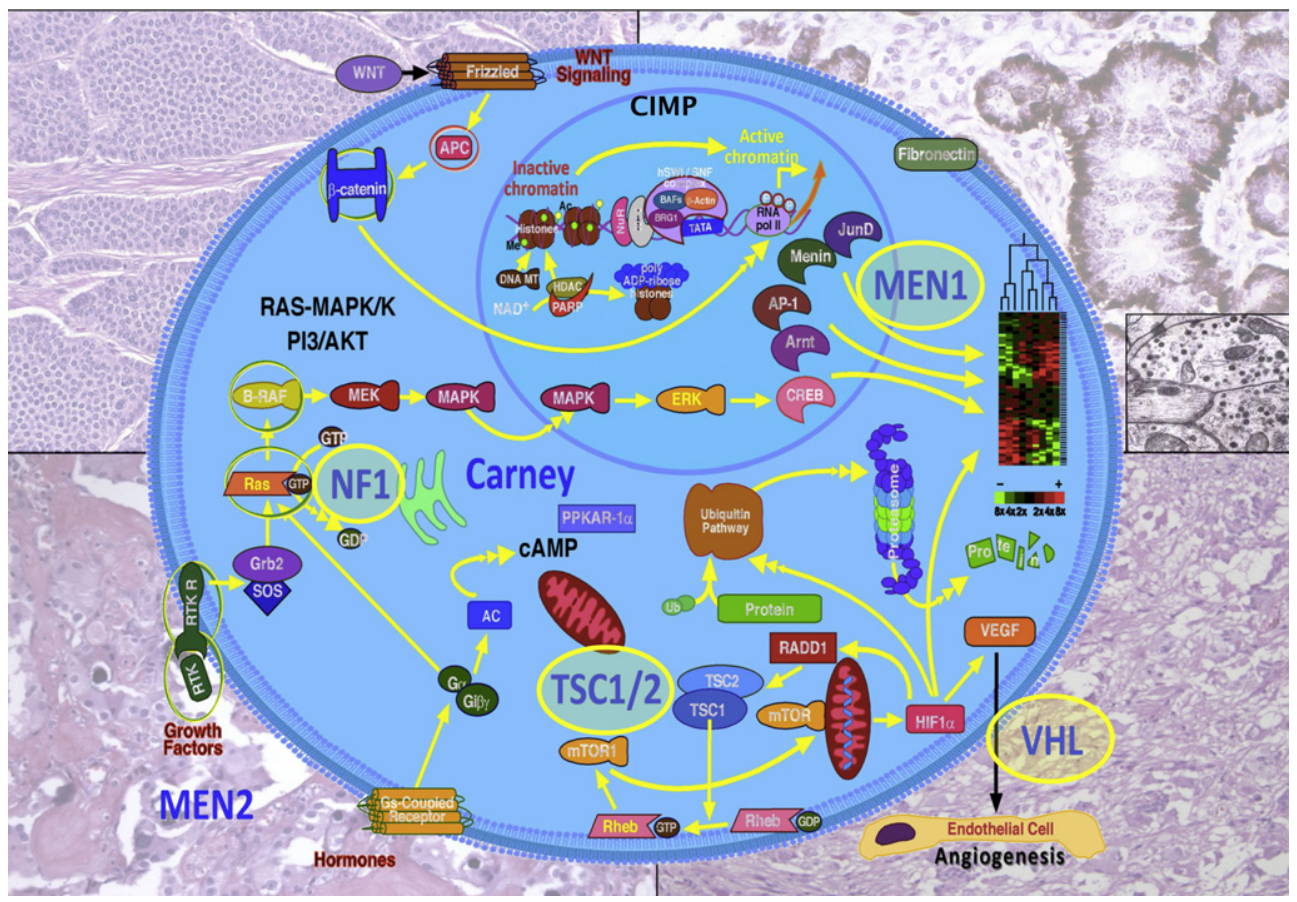

Figure 2 The general mechanisms of NETs include alterations of the WNT pathways, MAPK pathways, chromatin organization and angiogenesis. These cellular processes have key regulators that include known genes related with inherited syndromes with these neoplasms, such as multiple endocrine neoplasma type 1, von Hippel-Lindau disease, neurofibromatosis type 1 and tuberous sclerosis. The background reveals the spectrum of pathological changes in NET: a typical NET growth pattern, Grimelius positivity associated with secretory granules, psammomatous calcification of duodenal somatostatinomas, and the ganglionic differentiation of neural type NET of duodenum (gangliocytic paragangliomas). Multiple endocrine neoplasia (MEN) syndrome, type 1 is an autosomal dominant condition due to germline mutations of the MEN1 tumour suppressor gene. Menin interacts with a large number of proteins that are implicated in transcriptional regulation, genomic stability, cell division and cell-cycle control. MEN1 associated NETs display a wide variety of molecular abnormalities including chromosomal loss, chromosomal loss with duplication, mitotic recombination or point mutation of the wild-type allele. Like their sporadic counterparts, they exhibit inter- and intra-tumoural genetic heterogeneity indicating chromosomal instability. von Hippel-Lindau disease (VHL) is an autosomal dominant condition due to deletions or mutations in VHL tumour suppressor gene. This gene regulates the ubiquitination of the hypoxia-inducible factors HIF1 and 2, resulting in upregulation of angiogenic, growth and mitogenic factors including VEGF, PDGF $\beta$, TGF $\alpha$ and erythropoietin substances. Neurofibromatosis type I (NF1) is a relatively common disorder occurring in $1-4000-5000$ live births and is due to alterations in the NF1 gene. Neurofibromin acts as a tumour suppressor, affecting cell proliferation/growth and signalling by regulating the activation of p21 ras by its Ras GTPase protein-activating activity, binding microtubules, modulating adenylate cyclase activity, interacting with the cellular cytoskeleton and by interacting through a signalling pathway with tuberin (the TSC2 gene product) that regulates mTOR, a serine -threonine kinase that is involved in cell growth and proliferation. Alterations include nonsense, frameshift, and splice mutations, partial or complete deletions and translocation. Tuberous sclerosis (TS) is an autosomal dominant, neurocutaneous multisystem disorder that is due to mutations in one of two genes, the tuberous sclerosis complex 1 gene (TSC1) that encodes hamartin, or TSC2 that encodes tuberin. The two proteins produced by these genes dimerize and play a pivotal role in the phosphoinositide 3-kinase signalling pathway, and also are implicated in regulation of the small GTPase, rheb, which is involved in regulating mTOR activity.

interactions, resulting in unrestricted growth due to a stepwise accumulation of cooperative genetic alterations that affect key molecular pathways. The correlation of these molecular aspects with morphological changes is essential for better understanding of essential concepts as early neoplasms/precancerous lesions, progression/dedifferentiation, and intratumour heterogeneity. ${ }^{14}$ The GI-NET tumour biology is complex and results from the interaction of a myriad of factors that influence growth, differentiation, interaction with tumour environment, and secretion. In broad terms, two mechanisms of tumourigenesis apply: the $\mathrm{CpG}$ island methylator phenotype (CIMP) pathway, and chromosomal instability. ${ }^{15}$ The microsatellite instability pathway does not appear to be implicated to any significant degree in the molecular pathogenesis of GI-NET. Different genes are involved in the aetiology of each type of NET and different genetic abnormalities that include point mutations, gene deletions, DNA methylation, chromosomal losses and chromosomal gains may be involved. GI-NETs frequently show
$18 \mathrm{q}$ losses, rather than the $11 \mathrm{q}$ losses involving the MEN1 gene. MEN1 gene deletions are very rare in ileal and appendiceal NET, as are somatic MEN1 mutations. Additional genetic changes of GINETs concern the tumour suppressor gene CDKN2A (p16) and CTNNB1 ( $\beta$-catenin) as well as the CIMP pathway (Figure 2). GINETs frequently reveal $C D K N 2 A$ methylation, CTNNB1 mutations (WNT signalling pathway), and alterations related with CIMP pathways, rather than chromosomal instability. Furthermore, the foregut, midgut and hindgut NETs develop via different molecular pathways. For example, foregut NETs have frequent deletions and mutations of the MEN1 gene, whereas midgut NETs have losses of chromosomes 18, 11q and 16q, and hindgut NETs, express TGF $\alpha$ and the EGF receptor. ${ }^{15}$ In addition, in lung NETs, a loss of chromosome $3 p$ is the most frequent change, and TP53 mutations and chromosomal loss of 5 q21 are associated with more aggressive tumours and poor survival. The high frequency of chromosome 18 deletions strongly indicates a genetic alteration of importance in 


\section{Markers of neuroendocrine tumours}

\section{Marker}

Chromogranins and secretogranins

Neuroendocrine secretory protein 55 (NESP-55)

Synaptophysin

Synaptic vesicle protein 2 (SV2)

\section{Function}

- Major constituents of neuroendocrine secretory granules

- Precise functions are unknown, but it is likely that they have important roles in the packaging and processing of regulatory peptides, and they are cosecreted with other secretory granule constituents
- 241 - amino acid polypeptide located within large dense core secretory granules and it is the product of a genomically imprinted gene transcribed exclusively from the maternal allele

- Calcium-binding glycoprotein that is the most abundant integral membrane constituent of neuronal synaptic vesicles

- Present primarily in microvesicles, while the chromogranin proteins are present primarily in dense core secretory granules

- An integral membrane protein that initially was described in the central and peripheral nervous systems

\section{Subtypes/distribution}

- Include chromogranin A, chromogranin $B$, and secretogranin II (chromogranin C). All three proteins contain multiple dibasic residues that are sites for endogenous proteolytic cleavage to smaller peptides, some of which are biologically active

- Include pancreastatin, parastatin, vasostatin, chromostatin, and $\beta$-granin. The chromogranin proteins have demonstrated distinctive celland tissue-specific patterns of distribution, which generally are maintained in corresponding neoplasms

- Chromogranin A is the major granin present in gastric NETs and serotonin-producing NETs of the appendix and ileum. Chromogranin B and secretogranin II, on the other hand, predominate in hindgut NETs and neuroendocrine carcinomas that lack chromogranin A

- Its value lies in the ability to stain pancreatic NETs and pheochromocytomas, whereas GI tract NETs are negative

- Neuronal cells typically show a punctate pattern of staining corresponding to synaptic regions, while neuroendocrine cells typically feature a diffuse cytoplasmic staining pattern

- Present in a wide range of neuroendocrine cells of both neural and epithelial types, their corresponding NETs, normal and neoplastic adrenal cortical cells

- Present in a wide variety of normal neuroendocrine cells, including those of the gastrointestinal tract, pancreas, pituitary, and thyroid gland

\section{Comments}

- Positivity for chromogranins generally correlates with the extent of granularity as noted by electron microscopy. The cells and tumours with numerous and well-developed secretory granules exhibit intense staining for chromogranins, while paucigranular cells exhibit weak staining or may be completely non-reactive. In these instances, antibodies to other generic neuroendocrine markers (e.g. synaptophysin) may be positive
- The latest addition to the chromogranin family
- Tumours with abundant secretory granules typically exhibit intense chromogranin reactivity, while cells with sparse granules may be negative for chromogranin but often are positive for synaptophysin

- Antibodies to chromogranins and synaptophysin should be used as complementary reagents

- In the GI tract, SV2 is colocalized with different amines and peptides, including serotonin, somatostatin, gastrin, 
Table 3 (continued)

Marker

Hormones

Intermediate filaments

distinguish epithelial

type (cytokeratins)

from neural type

(neurofilament

triplet proteins).
Function

- The hormonal profiles of normal and neoplastic neuroendocrine cells can be established by immunohistochemical analysis using antibodies to the mature hormone or hormone precursors or by in situ hybridization using probes for their corresponding messenger RNAs (mRNAs). The latter approach is particularly useful for the distinction of de novo biosynthesis of hormones from their specific uptake by target cells or from non-specific uptake

\section{- Cytokeratins}

- NETs of the epithelial type typically are positive with broadspectrum cytokeratin (CK) antibodies, often demonstrating a dot-like pattern, corresponding to perinuclear accumulations of intermediate filaments for both CAM 5.2 (CK8 and CK18) and MNF116 (CK5, CK6, CK8, CK17, and CK19)

- In some instances, normal and neoplastic neuroendocrine cells of epithelial type may contain neurofilament proteins in addition to cytokeratins

- Neurofilament triplet protein

- Although NETs of the neural type generally are negative for cytokeratins. Neurofilament triplet proteins, which represent the major intermediate filament of normal and neoplastic neuroendocrine cells of neural type, are composed of heteropolymers of 3 different subunits

\section{Subtypes/distribution}

- In general, staining patterns for chromogranin A, synaptophysin, and SV2 are comparable in tumours, but hindgut NETs typically show positive staining for SV2, weak reactivity for synaptophysin, and absent staining for chromogranin A

- Immunohistochemical analysis has been a particularly important approach to establish the hormonal profiles of neuroendocrine tumours. The results of immunohistochemical and in situ hybridization studies have led to the observation that neuroendocrine cells in different tissues may produce identical or biosynthetically related products, thus hampering their usefulness as site-specific markers to determine the sites of origin of metastatic tumours of unknown primary sites

- In some instances, the differential expression of cytokeratin types is particularly useful for assessing the origins of metastatic neuroendocrine carcinomas of unknown primary sites. In this regard, CK20 is present in the vast majority of primary neuroendocrine carcinomas of the skin, while it is expressed in fewer than $10 \%$ of small-cell carcinomas of other primary sites of origin. Antibodies to CK19 are variably reactive in these tumours

- All 3 neurofilament subunits are phosphorylated in proportion to the molecular weights of each subunit. In general, the extent of staining for neurofilament triplet proteins in NETs of the neural type parallels their degree of differentiation, with neurons showing the most intense staining. While

\section{Comments}

cholecystokinin, and enteroglucagon

- GI tract and pancreatic endocrine tumours, medullary thyroid carcinomas, and pheochromocytomas consistently express SV2 reactivity. SV2 is a particularly valuable marker for hindgut NETs

- Despite this issue, immunohistochemical methods for the characterization of the hormonal profiles of endocrine tumours is of considerable value for establishing the malignant potential of certain tumour types

- A subset of epithelial NETs of the lung, gastrointestinal tract, pancreas, thyroid, and skin is positive for cytokeratins and neurofilament triplet proteins
- In some instances, the differen tial expression of neurofilament triplet proteins has, in fact, been related to the site of origin of NETs of the epithelial type in the gastrointestinal tract.

- Focal immunoreactivity has been observed in a small proportion of paragangliomas, particularly those arising in the 
Table 3 (continued)

Marker

Enzymes

\section{Function}

with molecular weights of 70 , 170 , and $195 \mathrm{kd}$.

- The enolases are the products of three genetic loci that have been designated alpha, beta, and gamma

\section{- Protein gene product 9.5} (PGP9.5) is a C-terminal hydrolase present in neurons and a variety of neuroendocrine cells. ${ }^{57,58}$
- A variety of endopeptidases and carboxypeptidases are essential for the formation of biologically active peptides from inactive precursors
- Histidine decarboxylase is an enzyme for decarboxylating Lhistidine to histamine

\section{Subtypes/distribution}

neurofilament triplet proteins are not present in all poorly differentiated NETs of the neural type, other types of neurofilaments such as peripherin and $\mathrm{Nf}$-66/alpha-internexin may be present.

- Non-neuronal enolase $(\alpha-\alpha)$ is present in fetal tissues, glial cells, and many nonneuroendocrine cells in the adult. Muscle enolase is of the $\beta$ - $\beta$ type, while the neuronal form of enolase has been designated $\gamma-\gamma$. Hybrid enolases are present in a wide variety of cell types. Although antibodies to neuron-specific enolase have a high sensitivity for the detection of neuroendocrine cells and their corresponding tumours, their specificity is low, particularly with polyclonal antisera

- The distribution of PGP9.5 generally is similar to that of neuron-specific enolase, and both markers show diffuse patterns of cytoplasmic reactivity that are unrelated to the type and content of hormone produced or to the degree of differentiation

- These enzymes are present in secretory granules and include the prohormone convertases (PC1/PC3, PC2) and carboxypeptidases $\mathrm{H}$ and $\mathrm{E}$
- It is expressed in various types of NE cells

\section{Comments}

cauda equina. Cytokeratin immunoreactivity also has been reported in up to $20 \%$ of primitive neuroectodermal tumours.

- Currently, polyclonal antibodies to neuron-specific enolase are used most effectively as a screening approach for the identification of normal and neoplastic neuroendocrine cells, but a confirmation of neuroendocrine differentiation must be established by more specific reagents
- Comparative studies have revealed that some NETs are positive for neuron-specific enolase and negative for PGP9.5, while other tumours show an opposite pattern of staining. Antibodies to PGP9.5 are particularly useful for the demonstration of neurons and cells with neuronal differentiation

- The distribution of these enzymes is restricted to neuroendocrine cells and their tumours, while other types of endocrine cells, such as thyroid follicular cells and adrenal cortical cells, are negative.

- NE cells and NETs with a neural phenotype contain a predominance of PC2, while epithelial neuroendocrine cells contain a predominance of $\mathrm{PC} 3$

- Recent findings have demonstrated a high expression in NETs, small-cell carcinomas of the lung, pheochromocytomas, and medullary thyroid carcinomas of the thyroid 
Table 3 (continued)

Marker

Somatostatin receptors (SSTR)

Membrane and adhesion molecules

\section{Function}

\section{- Catecholamine-synthesizing} enzymes [tyrosine hydroxylase (TH), dopamine-ß-hydroxylase (DBH), and phenylethanolamine- $N$-methyltransferase (PNMT)] has been demonstrated in midgut NETs and pheochromocytomas

- Somatostatin was identified as an important inhibitory hormone that binds to a family of five G-protein coupled receptors that inhibit adenylate cyclase activity

- Five somatostatin receptors have been identified

- The biochemical responses are believed to be mediated via SSTR2, whereas the antiproliferative effects are believed to be mediated via SSTR2 and SSTR5, while SSTR3 is thought to mediate induction of apoptosis. Anti-angiogenic and immunomodulatory effects have also been described

- CD56 or neural adhesion molecules (NCAM) include a family of glycoproteins that have key roles in cell binding, migration, differentiation, and proliferation. The members of the NCAM family are generated by alternative splicing of RNAs from a gene that is a member of the immunoglobulin supergene family. The molecules are modified posttranslationally by phosphorylation, sulfation, and glycosylation, and their binding properties are modulated by differential expression of polysialic acid

- The monoclonal antibody CD57 reacts with natural killer lymphocytes, myelin-associated glycoprotein, NCAMs, and a granule matrix constituent of chromaffin cells

- CD99, a transmembrane glycoprotein encoded by the MIC-2 gene, is widely expressed in a variety of normal cells,

\section{Subtypes/distribution}

- A significant subgroup of welldifferentiated NETs reveal TH (40\%), DBH (40\%\%), and PNMT (33\%), being associated with increased urinary excretion of catecholamines and metabolites in approximately half of them

- The ubiquitous SSTRs expression in $80-90 \%$ of neuroendocrine tumours, as shown by autoradiography, octreotide scintigraphy, and later by immunocytochemistry, led to the application of this inhibitor in the treatment of NETs almost 30 years ago

- The immunohistochemical profile of SSTRs in NETs may have clinical relevance for therapeutic decisions

- The neural cell adhesion molecules (NCAMs) although early studies indicated that NCAMs were restricted in distribution to the nervous system, more recent studies using antibodies to CD56 indicate a wider distribution, including cells of the neuroendocrine system, adrenal cortex, cardiac muscle, thyroid follicular epithelium, and proximal renal tubular epithelium

- Among NETs, CD57 is present in $100 \%$ of pheochromocytomas, $85 \%$ of extraadrenal paragangliomas, $85 \%$ of WD-NETs of diverse origin, and $50 \%$ of small-cell carcinomas

- CD99 immunoreactivity also is present in a subset of NETs of the epithelial type, including

\section{Comments}

- However, no clinically relevant association between enzyme expression and urinary excretion of catecholamines and metabolites is normally demonstrated

- Somatostatin analogue therapy has antiproliferative activity resulting in tumour stabilization, but without significant size reduction, and it is efficacious in reducing hormone excess

- The clinically available somatostatin analogues bind with high affinity to SSTR2 and SSTR5 and with lower affinity to SSTR3.

- NETs express several SSTRs, but up to $90 \%$ of serotonin- and gastrin-producing NETs of the distal jejunum and ileum, and about $60 \%$ of insulin-producing pancreatic NETs, are positive for SSTRs 2 and 5

- With the monoclonal antibody 735 (polysialic acid), they found positivity in $93 \%$ of small-cell carcinomas, $80 \%$ of large-cell neuroendocrine carcinomas, $67 \%$ of atypical carcinoids, and $59 \%$ of typical carcinoids. The median staining score was consistently higher in highgrade rather than in low-grade tumours. Thus, while CD56 is not absolutely specific for NETs, it remains a useful marker for these tumours and may be of use in the subclassification of some of them

- Results indicate that CD57 immunoreactivity is unreliable for the specific identification of NETS

- The value of CD99 immunostaining in the context of neuroendocrine tumours, 
Table 3 (continued)

Marker

Function

including cortical thymocytes, pancreatic endocrine cells, granulosa cells, Sertoli cells, and ependymal cells

\section{Subtypes/distribution}

small-cell carcinomas, pancreatic endocrine tumours, and WD-NETs of gastrointestinal and pulmonary origins
Comments

however, is its consistent negativity in neuroblastoma

Table 3

classical midgut NET tumourigenesis, apparently not involving the SMAD4/DPC4 gene. In this direction, sporadic and familial (autosomal dominant inherited tumour disease, familial ileal endocrine carcinoma, FIEC) ileal NET share clinical and molecular features, suggesting a common pathogenetic mechanism involved in tumour initiation; a putative FIEC tumour suppressor gene near the telomere of $18 \mathrm{q}$ has been proposed. Midgut NETs have revealed SDHD constitutional putative missense mutations (30\%) and loss of heterozygosity (LOH, 60\%) targeting both alleles, associated with $S D H D$ polymorphism as well, suggesting that the $S D H D$ gene is involved in the tumourigenesis of midgut NETs. Moreover, methylation frequencies of $R A R \underline{\beta}, C D H 1$ and $R A S S F 1 A$ genes increase with the severity of lung NETs. Thus the development and progression of NETs is associated with specific genetic abnormalities that indicate the likely involvement of different molecular pathways. Taken together, these data may explain why GI-NETs, depending on their site of origin and phenotype, differ in their biological behaviour and stress the importance of classifying NETs according to their localization and hormone production.

Recently it has been suggested that growth factors and their receptors are implicated in the proliferation of NETs, often acting in an autocrine or paracrine fashion. Several growth factors are thought to play a role including transforming growth factor $\beta$, insulin-like growth factor 1, vascular endothelial growth factor, platelet-derived growth factor, and fibroblast growth factors. The common oncogenes and tumour suppressor genes, including SRC, KRAS, MYC, FOS, JUN, TP53, RB1, PTEN, DPC4, CDKN2A/ p16, VHL, RET, BRAF, SMAD3 and the DNA mismatch repair genes, are not implicated in the molecular pathogenesis of these tumours, since mutations and/or $\mathrm{LOH}$ are either not found or are extremely rare in sporadic tumours.

Chromosomal instability has been implicated in tumour progression. Comparative genomic hybridization studies have shown losses of genetic material more often than gains, and amplifications are uncommon, ${ }^{15}$ and the number of genomic changes per tumour is associated with tumour size and disease stage, indicating that genetic alterations accumulate during tumour progression. Losses of chromosome 1 and $11 \mathrm{q}$ and gains on $9 \mathrm{q}$ appear to be early events that are already identified in small tumours. Metastases show prevalent gains of chromosomes 4 and 7 and loss of 21q, implying that these alterations may contribute to tumour dissemination.

NETs associated with syndromes are obviously associated with characteristic genetic abnormalities and four such syndromes exist: multiple endocrine neoplasia type 1 (MEN1 gene), von Hippel-Lindau disease (VHL gene), neurofibromatosis type 1 (NF1 gene), and tuberous sclerosis (TSC1 and TSC2 genes) (Figure 2). The MEN1 gene is involved in $20 \%-40 \%$ of sporadic
NETs as well. The role of NF1, VHL, and TSC1 and TSC2 in sporadic tumours is not well studied.

\section{Common problems in neuroendocrine neoplasms}

\section{Synchronic and metachronic neuroendocrine neoplasms - multifocal vs multicentric neoplasms}

Although clonality is considered the hallmark of neoplasms, the distinction between clonal origin and clonal expansion in tumours remains controversial. A priori, a monoclonal proliferation is assumed to be neoplastic, whereas a polyclonal lesion is thought to be reactive. However, there are many exceptions to this rule. Additionally, there is no consensus on the application of clonality markers. Clonal overgrowths represent the hallmark of neoplastic proliferations. Non-random genetic alterations can also be used to test clonal expansions and the clonal evolution of neoplasms, especially analyzing hypervariable deoxyribonucleic acid (DNA) regions from patients heterozygous for a given marker. ${ }^{16}$ These tests rely basically on the demonstration of $\mathrm{LOH}$ resulting from either hemizygosity (non-random interstitial DNA deletions) or homozygosity of mutant alleles observed in neoplasms. LOH analyses identify clonal expansions of a tumour cell population, and point to monoclonal proliferation when multiple and consistent $\mathrm{LOH}$ are demonstrated. Based on the methylation-related inactivation of one X-chromosome in female subjects, X-linked markers (e.g. androgen receptor gene) will provide clonality information using $\mathrm{LOH}$ analyses after DNA digestion with methylation-sensitive restriction endonucleases. Therefore, both non-X-linked and X-linked analyses give complementary information, related and not related to the malignant transformation pathway, respectively. ${ }^{17}$ Applied appropriately, these tools can establish the clonal evolution of tumour cell populations (tumour heterogeneity), identify early relapses, distinguish recurrent tumours from other metachronic neoplasms, and differentiate field transformation from metastatic tumour growths in synchronic and histologically identical neoplasms. ${ }^{18}$ The key elements for that distinction are: tumour natural history with particular attention to the relative timing between test conversion and clonal expansion, the lesion cell kinetic, and sample conditions. Studies based on allele ratio of genes involved in the transformation pathway must validate technique conditions to obtain reliable quantification methods able to detect clonal growths. Clonality analysis has been used to test malignant transformation and tumour progression, but the results must always be interpreted in view of the natural history of the neoplasm. The relationship between the molecular marker and the pathway of neoplastic transformation is essential, in particular, the relative timing between the positive conversion of 
the marker and the clonal expansion. ${ }^{19}$ Only simultaneous downregulated apoptosis and high proliferation result in selective kinetic advantage, dominant clone expansion, and unbalanced methylation patterns of androgen receptor alleles in neoplastic conditions.

GI-NETs are often multifocal, becoming clinically relevant to investigate whether multifocal intestinal NETs arise independently or whether they originate from a single clone with subsequent intramural spread. The clonality of intestinal NETs and the relationship between different tumour deposits of multiple intestinal NETs have shown controversial results. Initially, nonrandom X-chromosome inactivation patterns, compared with the background normal intestinal mucosal tissues, proved the monoclonal origin of human intestinal NETs. More interestingly, identical X-chromosome inactivation patterns were found in different NETs from each individual case, strongly indicating that multiple NETs of the small intestine were generated by metastasis of a primary tumour to different locations in the intestine, rather than being of multiple origin. Other study had revealed different $\mathrm{LOH}$ pattern for each tumour (46\%) with different $\mathrm{LOH}$ patterns among some of the coexisting tumours (38\%), whereas other coexisting tumours displayed the same allelic loss pattern. Only $8 \%$ cases showed the same $\mathrm{LOH}$ pattern in every individual tumour. X-chromosome inactivation analysis showed a discordant pattern of non-random X-chromosome inactivation in two of six informative cases and concordant pattern of non-random Xchromosome inactivation in the four remaining informative cases. These data suggest that some multifocal GI-NETs arise independently, whereas others originate as a single clone with subsequent local and discontinuous metastasis. An association of X-chromosome deletions with malignancy has already been found in gastric NETs. LOH was found in six of eight malignant PETs $(60 \%$ of the informative markers), but was infrequent in the nine benign ones $(4.5 \%)$. In contrast, although retention of heterozygosity was consistently observed in benign midgut NETs, LOH was infrequent in malignant NETs $(15 \%)$. No correlation was found between $\mathrm{LOH}$ and the ploidy status. These results indicate an association between X-chromosome $\mathrm{LOH}$ and malignancy in foregut NETs. The lack of such an association in midgut NETs suggests that different molecular mechanisms are involved in the progression of these two categories of NETs, which are otherwise considered to be closely related. These findings emphasize the need for carefully subdividing GI-NET according to their anatomical site of origin. ${ }^{20}$

Gastric NET associated with corporal (body of stomach) atrophic gastritis (CAG) is benign tumours developing as the final step of a hyperplastic precursor sequence (type I), for which the neoplastic nature has been assumed but never proved. Type III gastric NETs and NECs are malignant neoplasms without known precursor lesions. Clonality assays based on X-chromosome inactivation and $\mathrm{LOH}$ on the X-chromosome using three polymorphic markers (DXS989, DXS1003, DXS1192) have revealed monoclonal pattern in $88 \%$ of type I NETs. Extensive LOH of the X-chromosome involving at least two markers was found in all metastasizing NETs, but in none of the benign NETs. These results indicate that most type I NETs are true monoclonal neoplasms and that malignant evolution in gastric NETs is associated with extensive allelic deletion of one X-chromosome.

\section{Metastatic potential and prediction of primary site in NETs}

WD-NETs of the gastrointestinal tract, pancreas, and lung are histologically similar. Thus, predicting the site of origin of a metastasis is not possible on morphologic grounds. Current techniques to define neoplasia are limited but molecular genetic signatures can categorize tumours and provide biological rationale for predicting clinical behaviour, based on markers that have been implicated in tumourigenicity, metastasis, and hormone production. There have been several attempts to predict the origin based on the marker profiles from both immunohistochemical and gene expression analyses. A summary of these studies appears in Table $4 .^{21-25}$

Markers of aggressive behaviour are needed to distinguish lesions that are likely to be cured by surgery from those that will recur and metastasize. Overexpression of mRNA for the epidermal growth factor (EGF) and hepatocyte growth factor (HGF) receptors has been reported in a subpopulation of gastrinomas that exhibit more aggressive behaviour. ${ }^{26}$ Other markers that predict more aggressive malignancy include CD44s, which is more frequently negative in NETs with lymph node and/or visceral metastasis than in those without demonstrated metastasis $(P=0.030)$. NETs lacked HER2overexpression predictive of anti-HER2 response and KIT and PDGFRA activating mutations indicative of imatinib sensitivity. High EGFR aneusomy (20\% of all cases) and elevated EGFR copy number (39\%) were found, but few KRAS mutations associated with non-response to anti-EGFR therapy (3\%). Hsp90, TGFBR1, IGF1R, and SSTR5 exhibited highest levels of immunohistochemical staining in the largest percents of tumours. ${ }^{27}$ These results support further research into Hsp90, IGF1R, and EGFR as targets for developing new anticancer therapeutics for some NETs.

\section{Conclusions}

GI-NETs represent a relatively rare group of lesions that pose diagnostic and therapeutic challenges. The definition of malignancy is difficult and often impossible based on conventional histology, and the management of patients with lesions of uncertain malignant potential is controversial. These tumours are clinically and pathologically heterogeneous, for which it is critical to convey in diagnostic terminology both the type and the malignant potential of the particular neoplasm. This can be accomplished by the use of any of the classification schemes illustrated in Figure 1. The value of the term neuroendocrine is related to the fact that it connotes a particular phenotype or pattern of differentiation. Accordingly, the nomenclature of NETs can be addressed by using any of the following groups of terms: (1) WD-NET, WD-NEC, or PD-NEC; or (2) NEC (grades I-III); and by indicating in an explanatory note that this diagnostic terminology is equivalent to the standard diagnostic terminology (carcinoid, atypical carcinoid, small-cell carcinoma), when appropriate. Ultimately, the use of specific terms for these neoplasms remains a personal preference, but what is the most critical is the necessity that the terms will be understood by health professionals caring for patients and that the terms can be grouped and translated for epidemiologic studies. Once malignancy is established, they offer a unique target for specific therapies that may hold promise, but remain to be validated in large series and multiple centres. 


\section{Molecular signatures of GI-NET: Prediction of behaviour and primary origin}

\section{Gene Signature}

CDX-2, PDX-1, TTF-1, and NESP-55

CDX-2 (homoeobox gene essential for intestinal development and differentiation)

Melanoma antigen family D2 (MAGE-D2), metastasisassociated 1 (MTA1), nucleosome assembly protein 1-like (NAP1L1), Ki-67 (a marker of proliferation), survivin, frizzled homologue 7

(FZD7),

the Kiss1 metastasis

suppressor

(Kiss1), neuropilin 2 (NRP2),

and chromogranin $A(\mathrm{CgA})$

Chromosome 18 aberrations

Gain of chromosome 7

NAP1L1, MAGE-D2, and MTA1

\section{Prediction of behaviour}

Predict development of metastasis WD-NETs, WD-NECs, and PDNECs were classified with a specificity of $78 \%, 78 \%$, and $71 \%$, respectively PD-NECs were misclassified as either WD-NETs or PDNETS

Metastases were predicted in all cases with $100 \%$ sensitivity and specificity

Exclusively observed in metastases

Elevated expression in NEC and goblet cell mixed NEC in comparison with NET in appendicitis

\section{Prediction of location}

Pancreatic origin: NESP-55/

PDX-1 + ve, CDX-2/TTF-1

-ve: $97 \%$ specificity

Ileal origin: CDX-2 +ve, PDX-1/NESP-55/TTF-1 -ve, $97 \%$ sensitivity and $91 \%$ specificity

TTF-1 + ve was confined to pulmonary NETs (present in only about a third of cases) NESP-55 + ve restricted to pancreas and the adrenal medulla

Approximately $80 \%$ of $\mathrm{Gl}$ tract NETs are CDX-2 positive, especially those occurring in the ileum and appendix

Expression of CDX-2 in more than $50 \%$ of tumour cells was seen only in midgut NETs $(P<0.001)$

Define primary small intestine NETs

SI NETs overexpress the neoplasia-related genes NAP1L1 (mitotic regulation), MAGE-D2 (adhesion), and MTA1 (oestrogen antagonism)

Identified in both sporadic and in familial ileal NETs; $100 \%$ vs $38 \%$, respectively

Appendix NET/NEC

\section{Comments}

A panel of these four markers may be useful in predicting the primary site of metastatic WD-NEC

Midgut NETs and their metastases are distinct from foregut and hindgut carcinoids in that they express high levels of CDX-2 Foregut NETs (lung, stomach) tend to be CDX-2 negative

Complement traditional pathologic criteria Primary SI NETs could be differentiated from normal human EC cells with $100 \%$ specificity and $92 \%$ sensitivity

The ability to determine the malignant potential of these tumours and their propensity to metastasize provides a biological rationale for the management of NETs and may have prognostic utility Global expression profiles revealed no differentially expressed genes Correlated with solid growth pattern $(P<0.01)$, a histopathological feature that has previously been related to worse prognosis Differences in NALP1gene expression (decreased in goblet cell mixed NE carcinomas) provide molecular signatures to identify appendiceal NETs

(continued on next page) 


\begin{abstract}
Table 4 (continued)

\section{Gene Signature}

PAX8 (encode a family of transcription factors that regulate organogenesis and cell-lineage specification in multiple organ systems)
\end{abstract}

Xenin (25-amino acid peptide that appears to be specific to duodenal neuroendocrine cells)

Chromogranin A, MAGE-D2 (adhesion), and MTA1 (metastasis)

\section{Prediction of behaviour}

Expression of PAX8 has been reported associated with WHO category 1-3 (positive in $100 \%, 64 \%$, and $52 \%$ of tumours,

respectively)

PAX8-negative tumours

were more frequently

associated with liver metastases

MTA1 is a marker of tumour invasion

\author{
Ghrelin (28-amino acid \\ acylated \\ peptide seen in oxyntic \\ glands \\ of the gastric mucosa by \\ cells \\ different from $E C L$, \\ enterochromaffin, \\ or $D$ cells and probably of \\ the $X$-like type)
}

\section{Prediction of location}

Positive in the majority of duodenal and rectal NETs, and in a minor subset of appendiceal and gastric NETs

Absent in ileal and

pulmonary NETs

It has been shown that duodenal NETs (nonfunctional, gastrin- and somatostatin-producing tumours) show xenin expression, being other $\mathrm{GI}$ NETs negative Chromogranin A discriminates NETs from other gastric neoplasms Overexpression of MAGE-D2 and MTA1 differentiate Type III/IV from Type I/II NETs GISTs share similar expression patterns with Type III/IV NETs, but have decreased chromogranin $\mathrm{A}$ Expression has also been detected in neuroendocrine cells in the pancreas, pituitary, and heart Ghrelin-producing NETs have been documented in the stomach and intestine

\section{Comments}

PAX8 expression was not associated with patient age, gender, MIB1 index, or lymph node metastases
Provides a molecular basis to define gastric NETs, GISTs, or adenocarcinomas

Table 4

Molecular studies, including gene expression profiling, and immunohistochemical profiles will be critical for further characterizing individual NET types and for elucidating their interrelationships: clonal relationship of multifocal/multicentric neoplasms and prediction of origin for metastatic neoplasms.

\section{REFERENCES}

1 Williams ED, Sandler M. The classification of carcinoid tumours. Lancet 1963; 1: 238-9.

2 Pearse AG. The diffuse endocrine system and the implications of the APUD concept. Int Surg 1979; 64: 5-7.
3 Le Douarin MN, Kalcheim C, Crest TN. The neural crest. Cambridge: Cambridge Universtity Press, 1999.

4 Adameyko I, Lallemend F, Aquino JB, et al. Schwann cell precursors from nerve innervation are a cellular origin of melanocytes in skin. Cell 2009; 139: 366-79.

5 Cheng H, Leblond CP. Origin, differentiation and renewal of the four main epithelial cell types in the mouse small intestine. III. Enteroendocrine cells. Am J Anat 1974; 141: 503-19.

6 Yao JC, Hassan M, Phan A, et al. One hundred years after "carcinoid": epidemiology of and prognostic factors for neuroendocrine tumors in 35,825 cases in the United States. J Clin Oncol 2008; 26: 3063-72.

7 Modlin IM, Lye KD, Kidd M. A 5-decade analysis of 13,715 carcinoid tumors. Cancer 2003; 97: 934-59. 
8 Plockinger U, Rindi G, Arnold R, et al. Guidelines for the diagnosis and treatment of neuroendocrine gastrointestinal tumours. A consensus statement on behalf of the European Neuroendocrine Tumour Society (ENETS). Neuroendocrinology 2004; 80: 394-424.

9 DeLellis RA, Lloyd RV, Heitz PU, Eng C. World Health Organization classification of tumours. Pathology and genetics of tumours of endocrine organs. Lyon: IARC Press, 2004.

10 Kulke MH, Siu LL, Tepper JE, et al. Future directions in the treatment of neuroendocrine tumors: consensus report of the National Cancer Institute Neuroendocrine Tumor clinical trials planning meeting. I Clin Oncol 2011; 29: 934-43.

11 Rindi G, Kloppel G. Endocrine tumors of the gut and pancreas tumor biology and classification. Neuroendocrinology 2004; 80(suppl 1):12-5.

12 Rindi G, Kloppel G, Alhman H, et al. TNM staging of foregut (neuro) endocrine tumors: a consensus proposal including a grading system. Virchows Arch 2006; 449: 395-401.

13 Rindi G, Kloppel G, Couvelard A, et al. TNM staging of midgut and hindgut (neuro) endocrine tumors: a consensus proposal including a grading system. Virchows Arch 2007; 451: 757-62.

14 Diaz-Cano SJ. General morphological and biological features of neoplasms: integration of molecular findings. Histopathology 2008; 53: 1-19.

15 Perren A, Anlauf M, Komminoth P. Molecular profiles of gastroenteropancreatic endocrine tumors. Virchows Arch 2007; 451(suppl 1): S39-46.

16 Diaz-Cano SJ. Designing a molecular analysis of clonality in tumours. J Pathol 2000; 191: 343-4.

17 Diaz-Cano SJ. Clonality studies in the analysis of adrenal medullary proliferations: application principles and limitations. Endocr Pathol 1998; 9: 301-16.

18 Diaz-Cano SJ, Blanes A, Wolfe HJ. PCR techniques for clonality assays. Diagn Mol Pathol 2001; 10: 24-33.

19 Pozo-Garcia L, Diaz-Cano SJ. Clonal origin and expansions in neoplasms: biologic and technical aspects must be considered together. Am J Pathol 2003; 162: 353-4. author reply 354-355.

20 Pizzi S, D’Adda T, Azzoni C, et al. Malignancy-associated allelic losses on the X-chromosome in foregut but not in midgut endocrine tumours. J Pathol 2002; 196: 401-7.

21 Drozdov I, Kidd M, Nadler B, et al. Predicting neuroendocrine tumor (carcinoid) neoplasia using gene expression profiling and supervised machine learning. Cancer 2009; 115: 1638-50.

22 Jaffee IM, Rahmani M, Singhal MG, Younes M. Expression of the intestinal transcription factor $\mathrm{CDX} 2$ in carcinoid tumors is a marker of midgut origin. Arch Pathol Lab Med 2006; 130: 1522-6.

23 Kidd M, Modlin IM, Mane SM, Camp RL, Eick G, Latich I. The role of genetic markers-NAP1L1, MAGE-D2, and MTA1-in defining smallintestinal carcinoid neoplasia. Ann Surg Oncol 2006; 13: 253-62.

24 Kidd M, Modlin IM, Mane SM, et al. Utility of molecular genetic signatures in the delineation of gastric neoplasia. Cancer 2006; 106: 1480-8.

25 Modlin IM, Kidd M, Latich I, et al. Genetic differentiation of appendiceal tumor malignancy: a guide for the perplexed. Ann Surg 2006; 244: 52-60.

26 Peghini PL, Iwamoto M, Raffeld M, et al. Overexpression of epidermal growth factor and hepatocyte growth factor receptors in a proportion of gastrinomas correlates with aggressive growth and lower curability. Clin Cancer Res 2002; 8: 2273-85.
27 Gilbert JA, Adhikari LJ, Lloyd RV, et al. Molecular markers for novel therapies in neuroendocrine (carcinoid) tumors. Endocr Relat Cancer 2010; 17: 623-36.

\section{Practice points}

- Synoptic reporting is advocated in a format that incorporates histologic and immunohistochemical findings, together with the WHO classification.

- GI-NETs have been categorized on the basis of sites of origin, hormonal profiles, and associated syndromes into benign NETs, benign/low-grade malignant NETs, and high-grade NEC. An additional approach uses the term NEC for all NETs and divides them into three grades. In this system, grade I NEC includes carcinoids with typical morphologic features, while grade II NEC includes atypical carcinoids and related neoplasms, and grade III NEC includes both small-cell and large-cell carcinomas (Figure 1). This classification acknowledges the important fact that all NETs are potentially malignant. Specific NET patterns of malignancy are, to some extent, site-dependent.

- The distinction of WD-NETs from WD-NECs is based on size and site, the presence of local invasion, angioinvasion, and metastases. In addition, cytologic atypia, mitotic index, Ki67 proliferative rate, and patterns of hormone production are additional important parameters of the classification.

- The diagnosis of PD-NEC in this system is more straightforward, since these tumours often show small-cell features, high mitotic rates, and necrosis.

- Tumours of moderate degrees of differentiation, comparable to the atypical carcinoids of the lung, also may occur in the gastrointestinal tract, but at present, they are not categorized separately. It has been suggested that the presence of such atypical features be included in the histologic report to alert the clinicians of potentially more aggressive behaviour.

- It is recommended that the morphologic and functional status be recorded. These terms then are modified by the inclusion of the functional aspects of the tumours (e.g. gastrin-, or serotonin-producing), although it is worth reiterating that immunohistochemically detected peptides do not imply that the patient has clinical symptoms, nor does this finding imply that the tumour is functional. It should be borne in mind that most patients are not evaluated biochemically for the full spectrum of peptide products of NET, and that absence of recognizable clinical features may not necessarily reflect true lack of clinical function, and subtle clinical manifestations may be missed.

- A comment on the extent of invasion through the GI wall may be added, although this feature determines the WHO category that the tumour is placed into and may represent a degree of repetition. In the case of a syndromic NET, a comment on the associated syndrome may be added. 


\section{Research directions}

- Functional and location-specific studies are needed to improve the accuracy of prognosis.

- Both targeted and individualized therapies require more detailed genomic and expression profiling of NET to identify predictive tests.

- Robust molecular definitions of tumour progression and multicentricity vs multifocality need to be applied for a better stratification of patients and precise prognosis. 